\title{
Incisión Fúndica Uterina con Histerectomía y Ligadura de Arterias Hipogástricas para manejo de Placenta Previa con Acretismo: A propósito de un caso.
}

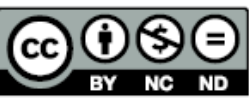

Este artículo está bajo una liencia de Creative Commons e tipo Reconocimiento - No comercial - Sin obras derivadas 4.0 International

1. Especialista en Ginecología y Obstetricia - Hospital General Docente Ambato

2. Profesor Auxiliar de Ginecología - Universidad Técnica de Ambato.

ORCID ID:

Yajaira Belalcázar

https:// orcid.org/0000-0003-0316-9971

Recibido: 05 - Sep - 2019

Aceptado: 09 - Oct - 2019.

Publicado: 01 - Dec - 2019.

Correspondencia: Yajaira Belalcázar

E-mail:dra_belalcazar@hotmail.com

Financiamiento: Reporte financiado con fondos propios del autor.

Conflictos de interés: El autor declara no poseer conflicto de interés.

\section{Resumen}

La placenta previa con acretismo es una patología con alta morbimortalidad que puede ser diagnosticada en el tercer trimestre del embarazo mediante estudios de imagen, lo que permite programar su resolución mediante cesárea. Se presenta un caso clínico de una paciente femenina de 40 años que cursaba un embarazo de 36,5 semanas con placenta previa oclusiva total con signos de acretismo determinados por ecografía y resonancia magnética nuclear. Considerando el riesgo de hemorragia se programó la cesárea mediante incisión fúndica del útero y al no desprenderse espontáneamente la placenta se realizó ligadura de arterias hipogástricas bilateral e histerectomía obstétrica, dicha cirugía se realizó sin complicaciones ni necesidad de transfusión sanguínea o ingreso de la paciente a terapia intensiva.

Palabras clave: placenta previa, placenta acreta, reporte de caso.
Forma de citar este artículo: Belalcazar Y. Incisión Fúndica Uterina con Histerectomía y Ligadura de Arterias Hipogástricas para manejo de Placenta Previa con Acretismo: A propósito de un caso. Rev Med Vozandes. 2019; 30(2): $52-56$

\section{Abstract}

\section{Uterine Fundic Incision with Histerectomy and} Hypogastric Artery Ligation for Placenta Previa Management with Acretism: About a Case.

The placenta previa with acretism is a pathology with high morbimortality that can be diagnosed in the third trimester of pregnancy by image, which allows to program its resolution by caesarean section. A clinical case of a 40 -year-old female patient presenting a 36.5-week pregnancy with total occlusive placenta with signs of accretion determined by ultrasound and resonance is presented, considering the risk of hemorrhage, a caesarean section was scheduled by means of a uterine fundal incision and at the placenta is not spontaneously detached, bilateral hypogastric artery ligation and obstetric hysterectomy are performed. This surgery is performed without complications or the need for blood transfusion or admission of the patient to intensive therapy

Keywords: :placenta previa, placenta accreta, case report. 


\section{Introducción}

La placenta previa constituye el $20 \%$ de las hemorragias del tercer trimestre, además es una causa importante de histerectomía obstétrica y necesidad de transfusión sanguínea materna, sobre todo cuando se asocia a acretismo, incrementando la morbi-mortalidad, razón por la cual los servicios de atención materno fetal deben contar con un protocolo de manejo de esta patología y entrenar al personal médico para su resolución de manera rápida y efectiva.

La cesárea programada en una paciente con placenta previa tiene como objetivo controlar las posibles complicaciones previstas para dicha patología, realizando una técnica quirúrgica con la incisión uterina lo más alejada posible del sitio de inserción placentaria y contar con un equipo multidisciplinario y capacitado, para resolver esta patología y reducir el sangrado, la necesidad de transfusión sanguínea, ingreso a terapia intensiva y muerte materna, entre otras morbilidades.

\section{Presentación del Caso}

Se presenta el caso de una paciente femenina de 40 años, mestiza, casada, instrucción superior, sin antecedentes patológicos de importancia, antecedentes ginecoobstétricos: menarquia 14 años, ciclos menstruales regulares de 3 días, inicio de vida sexual a los 19 años, tiene 2 gestas previas, 1 aborto espontáneo a las 8 semanas de gestación resuelto con legrado uterino instrumental y 1 cesárea por óbito fetal a las 35 semanas de gestación; actualmente cursa embarazo de 36,5 semanas por fecha de la última menstruación.

Paciente asintomática acude a control prenatal con ecografías que reportan placenta previa oclusiva total, al examen físico signos vitales estables, score mamá 0, abdomen gestante, feto único, cefálico, dorso derecho, frecuencia cardiaca fetal 141 latidos por minuto, sin actividad uterina. Examen especular cérvix cerrado, sin sangrado activo. Exámenes de laboratorio de ingreso: hematocrito de $41.5 \%$ y hemoglobina 13,1 g/dl.

Ecografía describe embarazo de 35,6 semanas, con placenta previa oclusiva total y signos de acretismo (figura 1)

Resonancia magnética obstétrica reporta placenta previa oclusiva total que cubre orificio cervical interno con signos de acretismo. (figura 2)

Con los datos mencionados se confirma el diagnóstico de embarazo de 36,5 semanas con placenta previa oclusiva total y acretismo placentario, en virtud de lo cual se programa la cesárea.

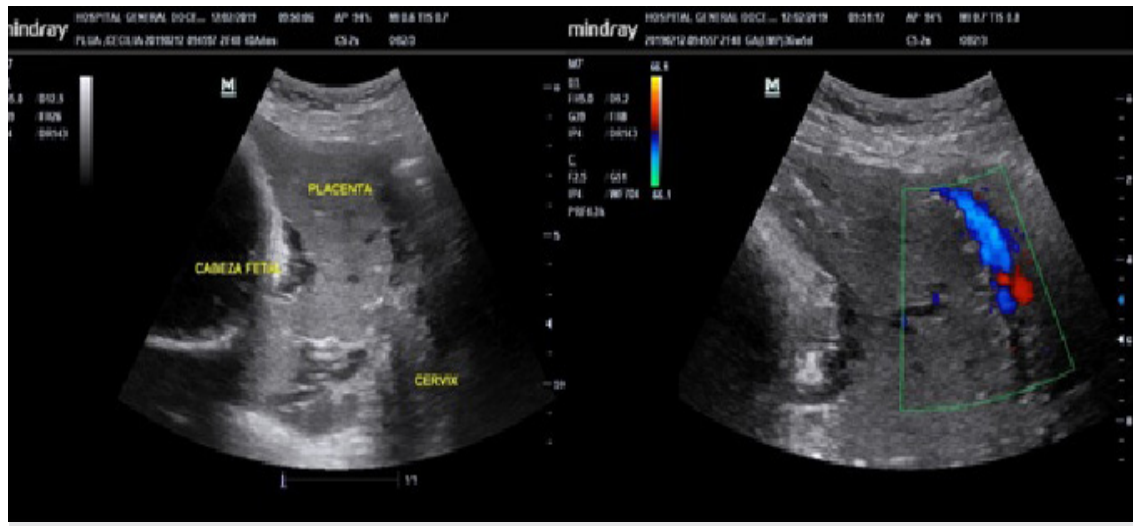

Figura 1. Placenta de implantación baja que hace contacto con el cérvix con hipervascularidad en la interfase útero-vejiga, sin evidenciarse adecuadamente pared vesical, además complejos venosos subplacentarios prominentes en relación con signos de acretismo placentario.

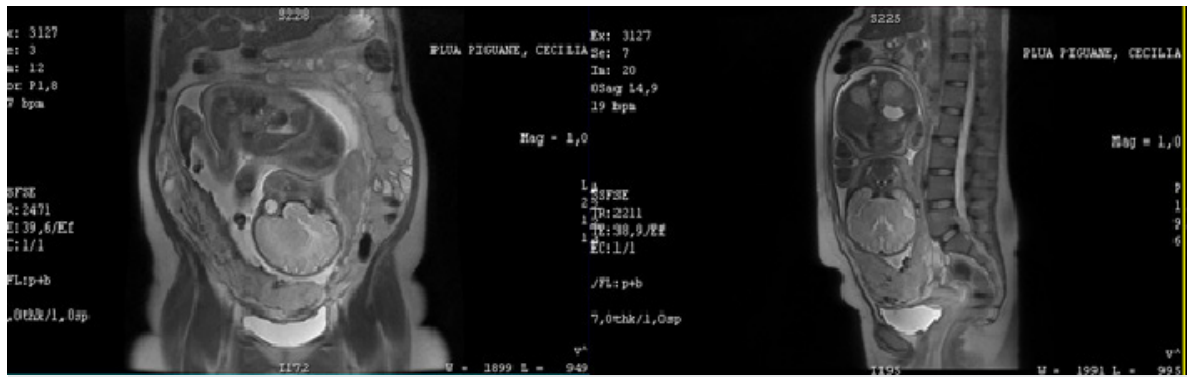

Figura 2. Resonancia magnética obstétrica: placenta previa oclusiva total que cubre orificio cervical interno, grosor $3.6 \mathrm{~cm}$, presenta finas bandas intraplacentarias hipointensas en secuencia T2 con saturación grasa, pequeños lagos venosos dispersos y pérdida de la diferenciación con el miometrio (línea hipointensa), infiltración al miometrio en su porción central. 

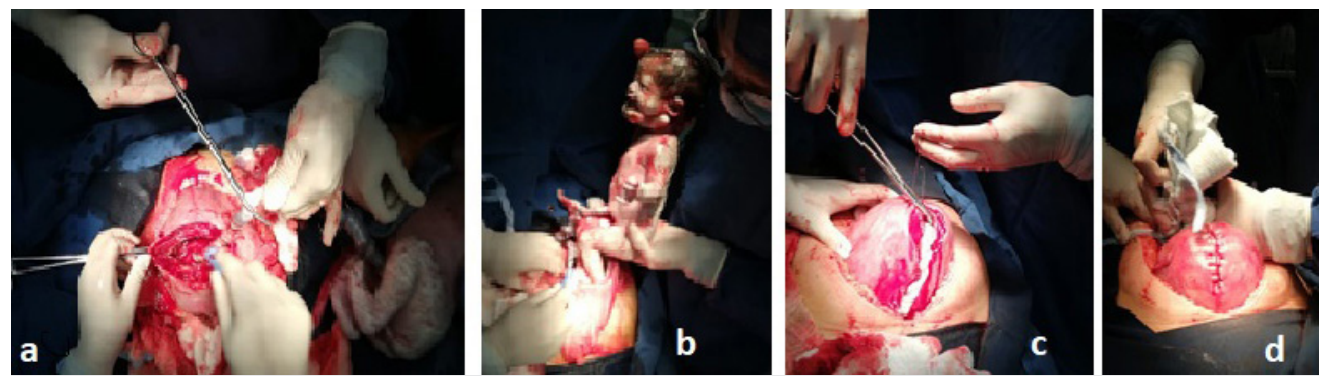

Figura 3. Cesárea.

a) Histerotomía fúndica

b) Extracción de RN

c) Clampeo y sección de cordón umbilical

d) Histerorrafia
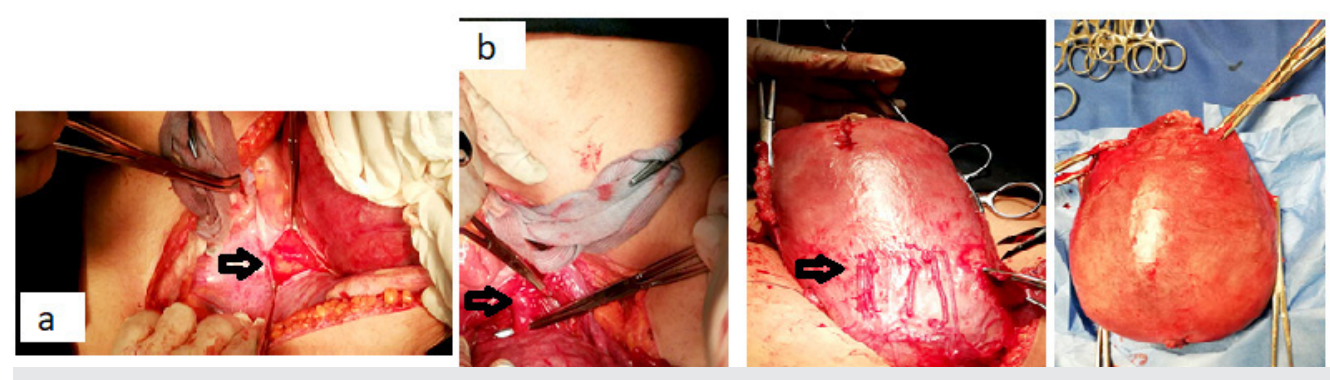

Figura 4.

a) Apertura de retroperitoneo

b) Ligadura de arterias hipogástricas bilateral

c) Visualización de signos de acretismo en segmento uterino

d) Histerectomía obstétrica

Durante el procedimiento quirúrgico se realiza una diéresis media supra e infraumbilical para apertura de la cavidad abdominal, se exterioriza el útero con el feto in situ para visualización del fondo uterino y se realiza la histerotomía mediante incisión fúndica vertical de aproximadamente $10 \mathrm{~cm}$.

Se obtiene un recién nascido vivo de sexo masculino, con escore APGAR 8-9, peso 2740 gramos, perímetro cefálico $33 \mathrm{~cm}$ y talla $48 \mathrm{~cm}$,(figura 3).

Durante el transquirúrgico evidenciamos vasos placentarios que invaden la serosa uterina y al no producirse el alumbramiento espontáneo después de la administración de oxitocina confirmamos el acretismo placentario. Posteriormente ligamos el cordón umbilical y realizamos una histerorrafia en un solo plano, sin remover la placenta para evitar una hemorragia masiva.

Después procedimos a ligar las arterias hipogástricas bilateral y efectuamos la histerectomía abdominal total, (figura 4) procedimiento sin complicaciones bajo anestesia general, con un sangrado transquirúrgico aproximado de $1800 \mathrm{ml}$.

El hemograma de control a las 6 horas del posquirúrgico muestra hematocrito de 34,9\% y hemoglobina de 11,2 g/dl, paciente con signos vitales estables y recuperándose.

El resultado histopatológico reporta: vellosidades coriales que invaden miometrio y llegan hasta la serosa, mayormente a nivel del segmento uterino, placenta pércreta a nivel de segmento, vellosidades coriales con congestión vascular acentuada.

\section{Discusión}

La placenta previa consiste en una localización anormal de la placenta, la misma que se encuentra en contacto con el orificio cervical interno $(\mathrm{OCl}) \mathrm{O}$ cubriéndole de forma parcial o total. ${ }^{(1,2)}$

No existe una clasificación universal para la placenta previa, sin embargo el criterio común es la relación de la placenta con el $\mathrm{OCl}$, es así que una de las clasificaciones más utilizadas, categoriza esta patología en: a) placenta previa oclusiva (llamada también previa completa o previa total) cuando la placenta cubre completamente el $\mathrm{OCl}(3,4,5)$, b) placenta previa no oclusiva, dentro de esta última se agrupa la placenta marginal, en la que la placenta es adyacente al borde del $\mathrm{OCl}$ sin sobrepasarlo y c) placenta de inserción baja, donde el borde placentario está a menos de $2 \mathrm{~cm}$ del $\mathrm{OCl}$. ${ }^{(5)}$ Actualmente se intenta definir solo 2 términos, el de placenta previa completa si cubre el $\mathrm{OCl}$ completamente o placenta previa parcial si el borde de la placenta no cubre completamente el $\mathrm{OCl}$ o si se encuentra a menos de $2 \mathrm{~cm}$ del mismo. ${ }^{(1,2,3,4)}$

El acretismo placentario es un desorden de placentación con daño en la interfase endometriomiometrio en la que hay adherencia anormal de la placenta al miometrio, por ausencia de la decidua basal y la falta de desarrollo de la membrana de Nitabuch, de acuerdo al grado de invasión del 
trofoblasto se encuentra la siguiente clasificación: placenta ácreta cuando las vellosidades coriales se adhieren al miometrio, placenta íncreta cuando las vellosidades penetran el miometrio y placenta pércreta cuando atraviesan el miometrio llegando hasta la serosa o más allá. $(3,10,11)$

Actualmente el principal factor de riesgo para acretismo placentario es la cirugía uterina y principalmente la cesárea por ausencia de reepitelización del tejido en el sitio de la cicatriz uterina permitiendo la invasión del trofoblasto al miometrio. ${ }^{(3,7)}$

Un metaanálisis mostró que la tasa de placenta previa aumenta con el número de partos por cesárea, con un 1\% después de 1 cesárea, 2,8\% después de 3 cesáreas y tan alto como $3,7 \%$ después de 5 cesáreas $(1,3,5)$

El sangrado de la placenta previa generalmente es indoloro y se produce frecuentemente en el tercer trimestre debido al crecimiento del segmento uterino inferior. $(1,10)$

Para determinar la ubicación de la placenta y su relación con el $\mathrm{OCl}$ se debe realizar una ecografía transvaginal en el segundo y tercer trimestre del embarazo, permitiendo además confirmar o no el diagnóstico de acretismo placentario.

En la ecografía transvaginal se observan múltiples lagunas vasculares dentro de la placenta, pérdida de la zona hipoecoica normal entre la placenta y el miometrio, disminución del grosor miometrial retroplacentario (menos de $1 \mathrm{~mm}$ ), anomalías de la interfaz de la serosa uterina y la vejiga. ${ }^{(3,5,7,12)}$

El uso de imágenes de flujo doppler puede facilitar el diagnóstico, el examen reporta flujo sanguíneo lacunar turbulento, aumento de la vascularización subplacentaria, brechas en el flujo sanguíneo miometrial y vasos que van de la placenta al margen uterino. ${ }^{(3,8.9)}$

Aún no está establecido que la resonancia magnética mejore el diagnóstico más allá de lo alcanzado con el eco trasvaginal. ${ }^{(3)}$

\section{Manejo}

La mayoría de las placentas previas identificadas en embarazos tempranos se resuelven con el avance de la gestación por lo que se debe realizar el seguimiento con ecografía transvaginal y doppler color pulsado. ${ }^{(2)}$

En caso de sangrado activo severo, hipotensión materna y patrón no reactivo de la frecuencia cardiaca fetal el parto por cesárea está indicado independientemente de la edad gestacional, pero si se estabilizan los signos vitales maternos, la frecuencia cardíaca fetal y el sangrado cede, se recomienda el manejo expectante en embarazos menores de 34 semanas. ${ }^{(2)}$

La hospitalización precoz en pacientes con placenta previa asintomática es todavía un tema controversial, en virtud del bajo riesgo de cesárea de emergencia. ${ }^{(2)}$

En embarazos que cursan con placenta previa no complicada se debe planificar la cesárea entre las 36 y 37,6 semanas sin documentación de madurez fetal por amniocentesis debido a que el riesgo asociado con sangrado severo o cesárea de emergencia es mayor que el riesgo de un neonato prematuro. ${ }^{(2,3)}$

La uteroinhibición puede ser útil en pacientes con actividad uterina, ya que las contracciones promueven la separación de la placenta y el sangrado.

Estudios observacionales (2-4), han determinado que el uso de uteroinhibidores prolonga el embarazo y permite mejorar el peso fetal. Por otro lado, la administración de corticoides no ha demostrado reducir los episodios de sangrado. La tocolisis está contraindicada en pacientes con sangrado activo. ${ }^{(2-4)}$

El parto por cesárea está indicado cuando hay evidencia ecográfica de una placenta previa completa y un feto viable. (7) El parto vaginal se puede considerar en raras circunstancias, como óbito fetal o feto no viable, siempre que la madre se encuentre hemodinámicamente estable. (2)

Cuando la placenta alcanza el $\mathrm{OCl}$, pero no lo cubre se ha planteado la posibilidad de parto vaginal considerando que la cabeza fetal tapona la placenta adyacente, evitando así la hemorragia, sin embargo, existe un alto riesgo de hemorragia intraparto por lo que sigue siendo la primera opción la cesárea programada. (2)

La cesárea de emergencia debe realizarse en caso de: a) frecuencia cardíaca fetal no reactiva y que no responde a la reanimación intra-útero, b) hemorragia que pone en riesgo la vida de la madre sin respuesta a las intervenciones habituales tales como transfusión, tocólisis y reposo y c) sangrado vaginal importante en embarazo mayor de 34 semanas. ${ }^{(2)}$

Durante la cesárea el cirujano debe evitar atravesar la placenta al entrar al útero, ya que la hemorragia fetal se relaciona con anemia neonatal, se debe disponer de 2 a 4 paquetes de sangre para transfusión y el instrumental necesario en caso de histerectomía. ${ }^{(2-3)}$

Si la placenta se encuentra anterolateral, se puede hacer una incisión vertical en el segmento uterino inferior en el lado opuesto a la placenta. Si la placenta se ubica a nivel de cuello uterino y se extiende al segmento anterior y posterior se debe realizar una incisión transversal o vertical alta. Cuando la incisión de la placenta es inevitable, se debe clampear rápidamente el cordón umbilical y entregar al recién nacido. ${ }^{(2)}$

La anestesia general está indicada en la cesárea de emergencia y con una paciente hemodinámicamente inestable y con un trazado de frecuencia cardíaca fetal no reactivo, sin embargo, la anestesia regional es una opción en pacientes con variables macro dinámicas estables (2)

Kotsuji F, et al. ${ }^{(6)}$, presentaron una serie de casos con incisión fúndica del útero y extracción de la placenta mediante visualización directa donde estimaron una pérdida de fluido incluido sangre y líquido amniótico de $1370 \mathrm{ml}$, sin necesidad de transfusión o ingreso a cuidados intensivos de ninguna paciente de un total de 34 participantes y se realizaron 19 histerectomías por imposibilidad de remover la 
placenta con confirmación de acretismo placentario por estudio histopatológico, (6) estos resultados son similares a los observados por Kayen $G$, et al. ${ }^{(13)}$, en su estudio de histerotomía con incisión vertical lejos de la inserción placentaria, y dejando la placenta in situ, protocolo adoptado de igual forma por nuestro servicio.

La técnica quirúrgica vascular integral avanzada (VIVA) con placenta adherida y feto in situ también describe la incisión fúndica del útero, no obstante, recomienda la ligadura de las arterias hipogástricas previo al nacimiento del feto.

En una serie de 16 casos en la ciudad de Querétaro se determinó que esta técnica reduce la estancia hospitalaria, la necesidad de transfusión sanguínea, el ingreso a terapia intensiva y la reintervención quirúrgica. ${ }^{(14)}$

La ligadura de arterias hipogástricas fue descrita por Howar Kelly en 1894, en los Estados Unidos; y desde ahí es una técnica poco utilizada por la falta de conocimiento y experiencia por parte del profesional Gineco-obstetra. Dicha ligadura en este caso es empleada de manera complementaria a la histerectomía con el fin de reducir el aporte sanguíneo al útero y con ello la hemorragia transquirúrgica. ${ }^{(15)}$

La programación de la cesárea en el presente caso por placenta previa oclusiva total con signos de acretismo determinados por imagen se realizó a las 36,5 semanas de gestación mediante histerotomía vertical fúndica para extracción del recién nacido sin atravesar la placenta y posterior realización de histerectomía obstétrica con la placenta in situ y previa ligadura de arterias hipogástricas como descrito en la literatura.

Con el procedimiento quirúrgico mencionado se observó la reducción de 2 puntos en el valor de hemoglobina materna postquirúrgica, sin necesidad de transfusión sanguínea ni ingreso a terapia intensiva como ocurre en la mayoría de los casos.

El procedimiento quirúrgico descrito se realiza como protocolo en el Servicio de Ginecología del Hospital General Docente
Ambato con el fin de reducir el sangrado transquirúrgico y con ello la morbilidad y mortalidad materna y neonatal. Se ha escogido esta técnica ya que permite realizar una incisión lo más alejada posible de la inserción placentaria, además de visualizar el desprendimiento o no de la placenta y efectuar una histerectomía con un sangrado más controlado.

El caso reportado servirá de base para elaborar un estudio con una serie de casos en el que se evalúe el beneficio del procedimiento comparado con otros estudios, además es necesario un seguimiento a largo plazo de las pacientes para evaluar los efectos posteriores de la cirugía, considerando sobre todo que la ligadura de arterias hipogástricas puede tener efectos colaterales.

\section{Conclusión}

La placenta previa y el acretismo placentario son patologías que presentan alta mortalidad y morbilidad debido a la hemorragia materna que pueden producir. En algunos casos con acretismo placentario se presenta la necesidad de realizar una histerectomía durante el parto o en el postparto. Por esta razón los servicios de atención materno fetal deben contar con personal entrenado y con experiencia en el manejo obstétrico de esta patología, así como disponer del apoyo de otros especialistas y servicios para realizar un manejo multidisciplinario. Teniendo en cuenta que ante la sospecha de placenta previa y acretismo placentario se debe programar el parto en un lugar que cuente con insumos, instrumental quirúrgico, personal capacitado y disponibilidad de banco de sangre.

\section{Referencias}

1. Bakker R. Placenta previa. Medscape. 2018. (Updated: Jan 08, 2018; cited: may 2019). Disponible: https://emedicine.medscape.com/ article/262063-overview

2. Lockwood Ch, Stieglitz K, Management of placenta previa, UpToDate, 2017. (Updated: Mar 03, 2017; cited: may 2019). Disponible: www.uptodate.com

3. Chahill A, Beigie R, Heine RP, Silver R, Wax J. Placenta Accreta Spetrum. Obstet Gynecol 2018;132:e259-75.

4. Lockwood Ch, Stieglitz K. Placenta previa: Epidemiology, clinical features, diagnosis, morbidity and mortality. UpToDate, 2019. (Updated: Jul 01, 2019; cited: Jul 2019). https:// www.uptodate.com/contents/placenta-previa-epidemiology-clinical-features-diagnosismorbidity-and-mortality/print

5. Perelló M, Mula R, López M. Anomalías placentarias (placenta previa, placenta accre ta y vasa previa) y manejo de la hemorragia de tercer trimestre. Institut Clínic de Ginecologia, Obstetrícia i Neonatologia, Hospital Clínic de Barcelona. 25/09/2012; 1-15
6. Kotsuji F, Nishijima K, Kurokawa T, Yoshida $Y$ Sekiya T, Banzai $M$, et al. Transverse uterine fundal incisionfor placenta praevia with accreta, involving the entire anterior uterine wall: a case series. BJOG 2013;120:1 144-1149.

7. Oppenheimer L. Diagnosis and Management of Placenta Previa. J Obstet Gynaecol Can of Placenta Previa.

8. Rac MW, Dashe JS, Wells CE, Moschos E, Mclntire DD, Twickler DM. Ultrasound predictors of placental invasion: the Placenta Accreta Index. Am J Obstet Gynecol. 2015 Mar;212(3):343.e1-7. doi: 10.1016/j. ajog.2014.10.022

. Wang Y, Gao Y, Zhao Y, Chong Y, Chen Y. Ultrasonographic diagnosis of severe placental invasion. J Obstet Gynaecol Res. 2018 Mar;44(3):448-455. doi:10.1111/jog.13531.

10. Silver R. Implantación anormal de la placenta Placenta Previa, Vasa Previa, y Placenta Ácreta. 2015 The American College of Obstetricians and Gynecologists, 2015;126:654-68. www.greenjournal.org
11. Veliz F, Nuñez F, Selman A. Acretismo placen tario: Un diagnóstico emergente. Abordaje quirúrgico no conservador. REV CHIL OBSTE GINECOL 2018; 83(5): 513 - 526.

12. Jauniaux $E$ Bhide $A$. Prenatal ultrasound diagnosis and outcome of placenta previa accreta after cesarean delivery: a systematic review and meta-analysis. Am J Obstet Gynecol. 2017 Jul;217(1):27-36.

13. Kayem G, Davy C, Goffinet F, Thomas C, Clément D, Cabrol D. Conservative versus extirpative management in cases of placenta accreta. Obstet Gynecol. 2004 Sep;104(3):531-6.

14. Villegas-Cisneros A, Ortega-Montoya C, García-López A. Técnica quirúrgica vascular integral avanzada (VIVA) aplicada en pacientes con placenta previa anormalmente adherida con feto in situ. Ginecol Obstet Mex. 2019 feb:87(1):36-45. https://doi.org/10.24245/ gom. v87il.2616

5. Espitia F, Zuluaga O, Orozco L. Ligadura de arterias hipogástricas en hemorragia postparto severa. CES MEDICINA. 2016; 30 (1). 